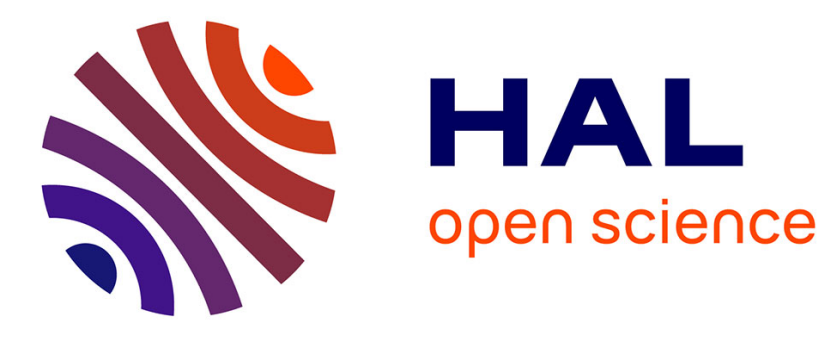

\title{
Voronoi-based UAVs Formation Deployment and Reconfiguration using MPC Techniques
}

Thomas Chevet, Cristina Stoica Maniu, Cristina Vlad, Youmin Zhang

\section{To cite this version:}

Thomas Chevet, Cristina Stoica Maniu, Cristina Vlad, Youmin Zhang. Voronoi-based UAVs Formation Deployment and Reconfiguration using MPC Techniques. International Conference on Unmanned Aircraft Systems, ICUAS'18, Jun 2018, Dallas, TX, United States. pp.9-14, 10.1109/ICUAS.2018.8453342 . hal-01768188

\section{HAL Id: hal-01768188 \\ https://hal.science/hal-01768188}

Submitted on 10 Sep 2018

HAL is a multi-disciplinary open access archive for the deposit and dissemination of scientific research documents, whether they are published or not. The documents may come from teaching and research institutions in France or abroad, or from public or private research centers.
L'archive ouverte pluridisciplinaire HAL, est destinée au dépôt et à la diffusion de documents scientifiques de niveau recherche, publiés ou non, émanant des établissements d'enseignement et de recherche français ou étrangers, des laboratoires publics ou privés. 


\title{
Voronoi-based UAVs Formation Deployment and Reconfiguration using MPC Techniques
}

\author{
Thomas Chevet*, Cristina Stoica Maniu*, Cristina Vlad*, and Youmin Zhang ${ }^{\dagger}$
}

\begin{abstract}
This paper presents a decentralized Voronoi-based linear model predictive control (MPC) technique for the deployment and reconfiguration of a multi-agent system composed of unmanned aerial vehicles (UAVs) in a bounded area. At each time instant, this area is partitioned into non-overlapping timevarying Voronoi cells associated to each UAV agent. The formation deployment objective is to drive the agents into a static configuration based on the Chebyshev center of each Voronoi cell. The proposed MPC-based formation reconfiguration algorithms allow not only faulty/non-cooperating agents to leave the formation, but also recovered/healthy agents to join in the current formation, while avoiding collisions. Simulation results validate the effectiveness of the proposed control algorithms.
\end{abstract}

\section{INTRODUCTION}

In the last decade, unmanned aerial vehicles (UAVs) have been the source of a growing interest coming from the fact that these vehicles can be used for various applications such as resource monitoring [1], forest fire surveillance [2], mapping [3] or rescue missions [4]. Control of UAVs is then an active research topic [5]. Some applications can use a formation of UAVs to distribute the work load. During such missions, the vehicles must avoid collisions with other vehicles while tracking autonomously a given reference.

Several control schemes have been studied over the years for UAVs formation control such as linear quadratic regulation [6], robust control [7], sliding mode control [8] or model predictive control (MPC) [9]. Nevertheless, the formation can evolve when one or several UAVs in the formation become non-cooperating leading to a faulty situation [10] or if one or more UAVs join in the formation. In a wider context, settheoretic methods [11] have been studied for the control of multi-agent systems [12], [13], with different applications. Area coverage problems have been actively studied [14], [15], however they are mainly focused on centroidal Voronoi configurations. Such a configuration frequently relies on the centers of mass of a Voronoi tessellation [16] which can be laborious to compute. A different approach considers the Chebyshev centers of the Voronoi tessellation [13], the computation of these centers being of reduced complexity.

In this paper, an open multi-agent system [17] (agents can join or leave the system) is considered where agents are quadrotor UAVs. The agents are deployed in formation in a

\footnotetext{
*Laboratoire des Signaux et Systèmes (L2S), CentraleSupélec-CNRSUniversité Paris-Sud, Université Paris-Saclay, 3, rue Joliot-Curie, 91192 Gif-sur-Yvette, France. E-mail: \{thomas.chevet, cristina.stoica, cristina.vlad\}@12s.centralesupelec.fr

$\dagger$ Department of Mechanical, Industrial and Aerospace Engineering, Concordia University, 1455 Blvd. de Maisonneuve, Montréal, QC H3G 1M8, Canada. E-mail: ymzhang@encs.concordia.ca
}

bounded area partitioned into a Voronoi tessellation. The formation objective is a static configuration based on the Chebyshev centers of the Voronoi tessellation. This paper proposes decentralized MPC techniques for formation deployment and reconfiguration of UAVs formation in case of incoming and outgoing agents. The contribution of this paper covers: 1) a MPC controller to deploy the UAVs formation into a static Chebyshev configuration; 2) the design of a barycenter-based MPC control algorithm for non-cooperating UAV agents to leave the formation or for agents to join in the formation. The proposed technique can be adapted to several types of agents, either aerial, terrestrial or aquatic, leading to a twodimensional approach for the formation. Moreover, several applications (surveillance, forest fire monitoring) require a constant altitude in order to avoid exposure or damage.

Section II describes the models considered in this paper. The mathematical tools for the control problem and the control objective of the system are presented in Section III. Section IV is dedicated to the design of the MPC algorithms for the control of UAVs formation. Section V details the simulation scenarios and the associated results. Concluding remarks and perspectives are drawn in Section VI.

Notation. In the following, $\mathbb{R}$ (resp. $\mathbb{N}$ ) is the set of the real numbers (resp. positive integers). The matrix $\mathbf{0}_{n \times m}$ is of size $n \times m$ and contains only zeros. If $n=m$, the notation becomes $\mathbf{0}_{n}$. The matrix $\boldsymbol{I}_{n}$ is the identity matrix of size $n$. The transpose of the $\boldsymbol{A}$ matrix is denoted by $\boldsymbol{A}^{\top}$. The notation $\|\mathbf{x}\|_{2}$ is the Euclidean norm of the vector $\mathbf{x}$ such that $\|\mathbf{x}\|_{2}^{2}=\mathbf{x}^{\top} \mathbf{x}$. The quadratic form $\|\mathbf{x}\|_{Q}$ is defined such that $\|\mathbf{x}\|_{\boldsymbol{Q}}^{2}=\mathbf{x}^{\top} \boldsymbol{Q} \mathbf{x}$. The notation $\boldsymbol{Q} \succ 0$ means that $\boldsymbol{Q}$ is a strictly positive definite matrix. The set of all integers from $m$ to $n$, with $m \leq n$, is denoted by $\{m, \ldots, n\}$. The sets $\mathscr{N}$, $\mathscr{N}_{f}$ and $\mathscr{N}_{i}$ are the sets of the integers indexing respectively all the considered agents, the agents steered into a formation and the neighbors of the agent indexed by $i \in \mathscr{N}_{f}$ while in formation $\left(\mathscr{N}_{f} \subseteq \mathscr{N} \subset \mathbb{N}, \mathscr{N}_{i} \subset \mathbb{N}\right)$. The cardinality of a set $\mathscr{S}$ is denoted by $|\mathscr{S}|$. The Minkowski sum of two sets $\mathscr{A}$ and $\mathscr{B}$ is the set $\mathscr{A} \oplus \mathscr{B}=\{a+b \mid a \in \mathscr{A}, b \in \mathscr{B}\}$.

\section{System MODELING}

This section presents the global multi-agent system and the mathematical model of each individual UAV. For the simplicity of the formulation, it is assumed that the formation is composed of homogeneous agents.

\section{A. Multi-agent system description}

The global system is a homogeneous multi-agent system $\Sigma$ composed of $|\mathscr{N}|$ agents characterized by their state vector 


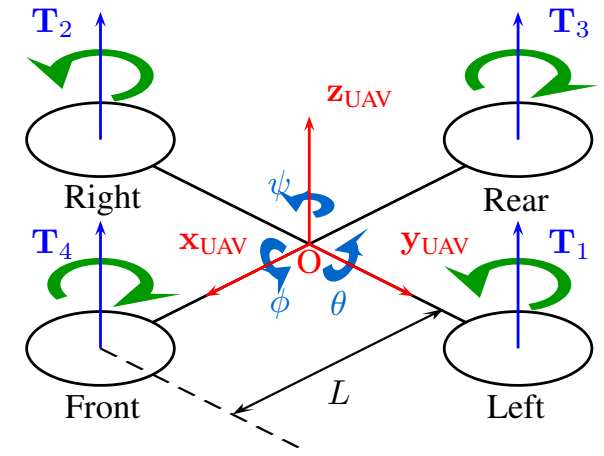

Fig. 1. Schematic representation of a quadrotor UAV.

$\mathbf{x}_{i} \in \mathbb{X} \subset \mathbb{R}^{n}$ and input vector $\mathbf{u}_{i} \in \mathbb{U} \subset \mathbb{R}^{m}$, with $i \in \mathscr{N}$.

Assumption 1. The number of agents $|\mathscr{N}|$ composing $\Sigma$ is time-varying.

All the agents use the continuous-time nonlinear dynamics:

$$
\begin{aligned}
& \dot{\mathbf{x}}_{i}(t)=\boldsymbol{f}\left(\mathbf{x}_{i}(t), \mathbf{u}_{i}(t)\right) \\
& \mathbf{y}_{i}(t)=\boldsymbol{C} \mathbf{x}_{i}(t)
\end{aligned}
$$

with the $i$-th agent's measured signals $\mathbf{y}_{i} \in \mathbb{Y} \subset \mathbb{R}^{p}$, the function $f: \mathbb{R}^{n} \times \mathbb{R}^{m} \rightarrow \mathbb{R}^{n}$, and the output matrix considered in the following to be $\boldsymbol{C}=\boldsymbol{I}_{12}$ yielding $\mathbb{Y}=\mathbb{X}$.

This continuous-time nonlinear dynamics can be linearized around an equilibrium point $\left(\overline{\mathbf{x}}_{i}, \overline{\mathbf{u}}_{i}\right)$, so that $\overline{\mathbf{y}}_{i}=\overline{\mathbf{x}}_{i}$, leading to the following continuous-time linear dynamics around this equilibrium point:

$$
\dot{\mathbf{x}}_{i}(t)=\boldsymbol{A}\left(\mathbf{x}_{i}(t)-\overline{\mathbf{x}}_{i}\right)+\boldsymbol{B}\left(\mathbf{u}_{i}(t)-\overline{\mathbf{u}}_{i}\right)
$$

where $\boldsymbol{A}$ and $\boldsymbol{B}$ are matrices of appropriate dimensions with $(\boldsymbol{A}, \boldsymbol{B})$ controllable and $(\boldsymbol{A}, \boldsymbol{C})$ observable with $\boldsymbol{C}=\boldsymbol{I}_{12}$. This linear model is used to design the controllers when addressing the formation related control issues.

In the following, in order to simplify the notation, the time dependency is dropped. Moreover, the variation terms around the equilibrium point $\mathbf{x}_{i}-\overline{\mathbf{x}}_{i}$ and $\mathbf{u}_{i}-\overline{\mathbf{u}}_{i}$ from (2) will be denoted by $\widetilde{\mathbf{x}}_{i}$ and $\widetilde{\mathbf{u}}_{i}$.

Assumption 2. The formation is composed by a part or all of the agents in $\Sigma$ evolving in a workspace $\mathbb{W} \subset \mathbb{X}$. The number of agents $\left|\mathscr{N}_{f}\right|$ composing the formation is time-varying.

\section{B. Dynamical model of the UAV}

Let us consider a set of homogeneous quadrotor UAVs composing the multi-agent system $\Sigma$. This type of UAV is actuated by four propellers driven by direct current (DC) motors placed as shown on Fig. 1. The state-space model of the UAV can be written by using the state vector $\mathbf{x}=$ $\left[\begin{array}{llllllllllll}x & y & z & \phi & \theta & \psi & v_{x} & v_{y} & v_{z} & \omega_{x} & \omega_{y} & \omega_{z}\end{array}\right]^{\top}$.

By using Lagrangian mechanics, the following state-space model of the UAV is derived [18]:

$$
\begin{aligned}
& \dot{x}=v_{x}, \quad \dot{y}=v_{y}, \dot{z}=v_{z} \\
& \dot{\phi}=\omega_{x}+\left(\omega_{y} \sin \phi+\omega_{z} \cos \phi\right) \tan \theta \\
& \dot{\theta}=\omega_{y} \cos \phi-\omega_{z} \sin \phi
\end{aligned}
$$

TABLE I

NOMENCLATURE AND NUMERICAL VALUES

\begin{tabular}{ll}
\hline$x, y, z$ & UAV's center of mass coordinates \\
$\phi, \theta, \psi$ & Pitch, roll and yaw angles \\
$v_{x}, v_{y}, v_{z}$ & Linear speed of the UAV \\
$\omega_{x}, \omega_{y}, \omega_{z}, i \in\{1, \ldots, 4\}$ & Angular speed of the UAV \\
$T_{i}=\left\|\mathbf{T}_{i}\right\|_{2}, i \in\{\}$ & Thrust of the $i$-th propeller \\
$u_{i}, i \in\{1, \ldots, 4\}$ & Supply voltage of the $i$-th rotor \\
$m=1.4 \mathrm{~kg}$ & UAV's mass \\
$I_{x}=I_{y}=0.03 \mathrm{~kg} \cdot \mathrm{m}^{2}$ & Moments of inertia along \\
$I_{z}=0.04 \mathrm{~kg} \cdot \mathrm{m}^{2}$ & $x, y$ and $z$ directions \\
$L=0.25 \mathrm{~m}$ & Arm's length \\
$C=1 \mathrm{~m}$ & Thrust to moment ratio \\
$K=120 \mathrm{~N} \cdot \mathrm{V}^{-1}$ & Motor gain \\
$\tau_{m}=5 \mathrm{~ms}$ & Motor mechanical time constant \\
$g=9.81 \mathrm{~m} \cdot \mathrm{s}^{-2}$ & Gravitational acceleration \\
$T_{s}=0.2 \mathrm{~s}$ & Sampling time \\
\hline &
\end{tabular}

$$
\begin{aligned}
\dot{\psi} & =\omega_{y} \frac{\sin \phi}{\cos \theta}+\omega_{z} \frac{\cos \phi}{\cos \theta} \\
m \dot{v}_{x} & =F_{t}(\cos \phi \sin \theta \cos \psi+\sin \phi \sin \psi) \\
m \dot{v}_{y} & =F_{t}(\cos \phi \sin \theta \sin \psi+\sin \phi \cos \psi) \\
m \dot{v}_{z} & =F_{t} \cos \phi \cos \theta-g \\
I_{x} \dot{\omega}_{x} & =\left(I_{y}-I_{z}\right) \omega_{y} \omega_{z}+\tau_{x} \\
I_{y} \dot{\omega}_{y} & =\left(I_{z}-I_{x}\right) \omega_{x} \omega_{z}+\tau_{y} \\
I_{z} \dot{\omega}_{z} & =\left(I_{x}-I_{y}\right) \omega_{x} \omega_{y}+\tau_{z} .
\end{aligned}
$$

The UAV's input $\left[\begin{array}{llll}F_{t} & \tau_{x} & \tau_{y} & \tau_{z}\end{array}\right]^{\top}$ can be expressed as a function of the propellers' thrust [19]:

$$
\left[\begin{array}{c}
F_{t} \\
\tau_{x} \\
\tau_{y} \\
\tau_{z}
\end{array}\right]=\left[\begin{array}{cccc}
1 & 1 & 1 & 1 \\
L & -L & 0 & 0 \\
0 & 0 & L & -L \\
-C & -C & C & C
\end{array}\right]\left[\begin{array}{c}
T_{1} \\
T_{2} \\
T_{3} \\
T_{4}
\end{array}\right]=\boldsymbol{B}_{1} \mathbf{T} .
$$

The time constant $\tau_{m}$ of the rotors' DC motors is negligible compared to the sampling period. Thus, the propeller's thrust is linked to its supply voltage by the first order relation [20]:

$$
T_{i}=K u_{i}, \text { with } i \in\{1, \ldots, 4\},
$$

the vector $\mathbf{u}=\left[\begin{array}{llll}u_{1} & u_{2} & u_{3} & u_{4}\end{array}\right]^{\top}$ being the input signal of the model. All the parameters mentioned above are gathered in Table I.

\section{Model linearization}

When in hovering state, the UAV's state vector is $\overline{\mathbf{x}}=$ $\left[\begin{array}{llll}\bar{x} & \bar{y} & \bar{z} & \mathbf{0}_{1 \times 9}\end{array}\right]^{\top}$. Thus, the supply voltage of each rotor is $\bar{u}_{i}=\frac{m g}{4 K}$, with $i \in\{1, \ldots, 4\}$.

The nonlinear model (3)-(14) is linearized around the equilibrium point $\overline{\mathbf{x}}$ leading to a linear state-space model around this point:

$$
\dot{\widetilde{\mathbf{x}}}=\left[\begin{array}{cccc}
\mathbf{0}_{3} & \mathbf{0}_{3} & \boldsymbol{I}_{3} & \mathbf{0}_{3} \\
\mathbf{0}_{3} & \mathbf{0}_{3} & \mathbf{0}_{3} & \boldsymbol{I}_{3} \\
\mathbf{0}_{3} & \boldsymbol{A}_{1} & \mathbf{0}_{3} & \mathbf{0}_{3} \\
\mathbf{0}_{3} & \mathbf{0}_{3} & \mathbf{0}_{3} & \mathbf{0}_{3}
\end{array}\right] \widetilde{\mathbf{x}}+\left[\begin{array}{l}
\mathbf{0}_{8 \times 4} \\
K \boldsymbol{B}_{1}
\end{array}\right] \widetilde{\mathbf{u}}
$$

with $\boldsymbol{B}_{1}$ given in (13) and $\boldsymbol{A}_{1}=\left[\begin{array}{ccc}g \sin \psi & g \cos \psi & 0 \\ -g \cos \psi & g \sin \psi & 0 \\ 0 & 0 & 0\end{array}\right]$. 
Assumption 3. The UAV is moving with a constant altitude $z=\bar{z}$ and a constant yaw angle $\psi=0$ during the entire flight for the model to be linear time invariant.

The following constraints are imposed on the state variables and are bounds of $\mathbb{X}$ :

$$
\begin{aligned}
|\phi|,|\theta| & \leq \frac{\pi}{12} \mathrm{rad} & \text { and } & & |\psi| & \leq 0.01 \mathrm{rad} \\
\left|v_{x}\right|,\left|v_{y}\right| & \leq 5 \mathrm{~m} \cdot \mathrm{s}^{-1} & \text { and } & & \left|v_{z}\right| & \leq 0.1 \mathrm{~m} \cdot \mathrm{s}^{-1} .
\end{aligned}
$$

If these inequalities hold, it is considered that the linearized model holds during the entire flight. This model will be used to design a controller in Section IV.

\section{Problem Formulation}

This section presents mathematical objects constructed on the workspace $\mathbb{W}$ and the main goal of the global system $\Sigma$ based on these objects.

\section{A. Voronoi tessellation}

Assumption 4. The workspace $\mathbb{W}$ is convex and its restriction to the first two directions of the state space, denoted as $\mathscr{W}$, is a bounded convex polytope.

The set $\mathscr{W}$ is a subset of $\mathbb{R}^{2}$ equipped with the Euclidean norm $\|\cdot\|_{2}$. If $\left|\mathscr{N}_{f}\right|$ agents are considered in $\mathscr{W}$, it can be partitioned in $\left|\mathscr{N}_{f}\right|$ Voronoi cells such that:

$$
\begin{aligned}
& \mathscr{W}=\bigcup_{i \in \mathscr{N}_{f}} \mathbb{V}_{i}, \text { with } \mathbb{V}_{i} \cap \mathbb{V}_{j}=\emptyset, \forall i, j \in \mathscr{N}_{f}, i \neq j \\
& \mathbb{V}_{i}=\left\{\mathbf{w} \in \mathscr{W} \mid\left\|\mathbf{x}_{i}^{r}-\mathbf{w}\right\|_{2} \leq\left\|\mathbf{x}_{j}^{r}-\mathbf{w}\right\|_{2}, \forall j \neq i\right\}
\end{aligned}
$$

with $\mathbf{x}_{i}^{r}$ and $\mathbf{x}_{j}^{r}$ the first two coordinates of the state vector of the $i$-th and $j$-th agents $\mathbf{x}_{i}, \mathbf{x}_{j} \in \mathbb{W}$. One cell is then the intersection of $\left|\mathscr{N}_{f}\right|-1$ bounded convex polytopes. The Voronoi cells are then bounded convex polytopes [21].

In the following, the $\mathscr{H}$-representation [22] of polytopes such as the Voronoi cell or the restricted workspace $\mathscr{W}$ will be used. If $\mathscr{P}$ is a polytope in $\mathbb{R}^{n}$ delimited by $m$ hyperplanes, then its $\mathscr{H}$-representation is given by:

$$
\mathscr{P}=\left\{\mathbf{x} \in \mathbb{R}^{n} \mid \boldsymbol{H}_{\mathscr{P}} \mathbf{x} \leq \boldsymbol{\Theta}_{\mathscr{P}}\right\}
$$

with $\boldsymbol{H}_{\mathscr{P}} \in \mathbb{R}^{m \times n}$ and $\boldsymbol{\Theta}_{\mathscr{P}} \in \mathbb{R}^{m}$.

The agents are equipped with sensors allowing them to know the position of their neighbors. They are then able to compute their own Voronoi cell. Because of Assumption 2, the number of agents in formation $\left|\mathscr{N}_{f}\right|$ and the Voronoi tessellation are time-varying.

\section{B. Chebyshev center}

The Chebyshev center $\mathbf{c}_{i}^{c}$ of $\mathbb{V}_{i}$, with $i \in \mathscr{N}_{f}$, is the center of the largest ball $\mathscr{B}_{i}=\left\{\mathbf{w} \in \mathbb{V}_{i} \mid\left\|\mathbf{w}-\mathbf{c}_{i}^{c}\right\|_{2} \leq r_{i}\right\}$ lying in $\mathbb{V}_{i}$. Here, $r_{i}$ designates the radius of the ball $\mathscr{B}_{i}$. The lines of $\boldsymbol{H}_{\mathbb{V}_{i}}$ and $\boldsymbol{\Theta}_{\mathbb{V}_{i}}$ are $\boldsymbol{h}_{\mathbb{V}_{i}}^{j}$ and $\theta_{\mathbb{V}_{i}}^{j}$, with $j \in\{1, \ldots, m\}$. The values of $\mathbf{c}_{i}^{c}$ and $r_{i}$ are then obtained by solving the linear optimization problem [23]:

$$
\begin{array}{ll}
\underset{\mathbf{c}_{i}^{c}, r_{i}}{\max } & r_{i} \\
\text { s.t. } & r_{i} \geq 0, \\
& \theta_{\mathbb{V}_{i}}^{j} \geq \boldsymbol{h}_{\mathbb{V}_{i}}^{j} \mathbf{c}_{i}^{c}+\left\|\boldsymbol{h}_{\mathbb{V}_{i}}^{j}{ }^{\top}\right\|_{2} r_{i}, j \in\{1, \ldots, m\} .
\end{array}
$$

Assumption 5. The restricted workspace $\mathscr{W}$ and the number of agents in the formation are given such that the Chebyshev center of each cell $\mathbb{V}_{i}$, with $i \in \mathscr{N}_{f}$, is unique.

A time-varying Voronoi tessellation leads to time-varying Chebyshev centers. The choice of the Chebyshev center is motivated by the simplicity of the linear optimization problem to be solved. Moreover, the Chebyshev center is defined as the farthest point from all sides of its cell, which minimizes the collision risk between the agents when they track this center.

Moreover, in [13], the formation converges towards a configuration where the agents' positions coincide with their associated Chebyshev centers with a null control signal when steered with a decentralized full state-feedback control law. Given Assumption 5 and the fact that the full state-feedback control law can easily be replaced by a MPC controller, the choice of the Chebyshev center is then justified.

\section{Control objective of the multi-agent system}

A multi-agent system $\Sigma$ is composed by $|\mathscr{N}|$ agents, indexed by integers $i \in \mathscr{N}$. As mentioned in Assumption 2, a subset of these agents $\mathscr{N}_{f} \subseteq \mathscr{N}$ evolves into a workspace $\mathbb{W} \subset \mathbb{X}$. The agents in $\mathscr{N}_{f}$ are being steered into a formation.

The first objective is the deployment of the agents controlled independently towards a static configuration in $\mathbb{W}$, where the output of each agent is the extended Chebyshev center $\widetilde{\mathbf{x}}_{i}^{c}=\left[\begin{array}{ll}\mathbf{c}_{i}^{c} & \mathbf{0}_{1 \times 10}\end{array}\right]^{\top}$, with $\mathbf{c}_{i}^{c}$ the Chebyshev center of the Voronoi cell $\mathbb{V}_{i}$. The second control objective consists in reconfiguring the formation whenever a non-cooperating agent leaves the formation lying inside $\mathbb{W}$. Finally, the reconfiguration has to be handled in case of incoming healthy agents being in $\mathbb{Y}$ but outside $\mathbb{W}$.

\section{Controller Design}

The main results of this paper are presented within this section: three decentralized linear MPC techniques for the formation deployment and the formation reconfiguration both for outgoing agents and for incoming agents.

\section{A. Decentralized formation deployment}

The decentralized formation deployment control algorithm is meant to steer each agent into the formation described in Section III-C. To this aim, the continuous-time linear model (15) is sampled with the sampling period $T_{s}$ leading to the discrete-time linear time invariant dynamics:

$$
\widetilde{\mathbf{x}}(k+1)=\boldsymbol{F} \widetilde{\mathbf{x}}(k)+\boldsymbol{G} \widetilde{\mathbf{u}}(k) .
$$

In the following, this model is used for all the agents, the variables $\widetilde{\mathbf{x}}$ and $\widetilde{\mathbf{u}}$ being indexed by $i \in \mathscr{N}$.

The control signal $\widetilde{\mathbf{u}}_{i}(k)$ for the agent $i \in \mathscr{N}_{f}$ is then obtained by solving the convex optimization problem, derived from the optimization problem [24, Chapter 4, p. 85]: 


$$
\begin{array}{ll}
\min _{\widetilde{\mathbf{u}}_{i}} \sum_{l=0}^{N_{p}-1}\left(\left\|\widetilde{\mathbf{x}}_{i}(k+l+1)-\widetilde{\mathbf{x}}_{i}^{\mathrm{obj}}(k)\right\|_{\boldsymbol{Q}}\right. \\
& +\left\|\widetilde{\mathbf{u}}_{i}(k+l)-\widetilde{\mathbf{u}}_{i}^{\mathrm{obj}}(k)\right\|_{\boldsymbol{R}_{1}} \\
& \left.+\left\|\widetilde{\mathbf{u}}_{i}(k+l)-\widetilde{\mathbf{u}}_{i}(k+l-1)\right\|_{\boldsymbol{R}_{2}}\right) \\
& +\left\|\widetilde{\mathbf{x}}_{i}\left(k+N_{p}\right)-\widetilde{\mathbf{x}}_{i}^{\mathrm{obj}}(k)\right\|_{\boldsymbol{P}} \\
\text { s.t. } \quad \widetilde{\mathbf{x}}_{i}^{\mathrm{obj}}(k)=\boldsymbol{F} \widetilde{\mathbf{x}}_{i}^{\mathrm{obj}}(k)+\boldsymbol{G} \widetilde{\mathbf{u}}_{i}^{\mathrm{obj}}(k), \\
\widetilde{\mathbf{x}}_{i}(k+l+1)=\boldsymbol{F} \widetilde{\mathbf{x}}_{i}(k+l)+\boldsymbol{G} \widetilde{\mathbf{u}}_{i}(k+l), \\
\widetilde{\mathbf{x}}_{i}(k+l+1) \in \mathbb{W}, \\
\widetilde{\mathbf{u}}_{i}(k+l) \in \mathbb{U}, \\
\widetilde{\mathbf{x}}_{i}\left(k+N_{p}\right) \in \mathbb{V}{ }_{i}^{\lambda}(k) .
\end{array}
$$

In the cost function (23a), the weighting terms $\boldsymbol{Q}, \boldsymbol{R}_{1}, \boldsymbol{R}_{2} \succ$ 0 are diagonal matrices. The matrix $\boldsymbol{P}$ is obtained by solving the algebraic Riccati equation [25]:

$$
\begin{aligned}
\boldsymbol{P}= & \boldsymbol{F}^{\top} \boldsymbol{P F}+\boldsymbol{Q} \\
& -\boldsymbol{F}^{\top} \boldsymbol{P} \boldsymbol{G}\left(\boldsymbol{R}_{1}+\boldsymbol{G}^{\top} \boldsymbol{P} \boldsymbol{G}\right)^{-1} \boldsymbol{G}^{\top} \boldsymbol{P F} .
\end{aligned}
$$

The objective point $\widetilde{\mathbf{x}}_{i}^{\text {obj }}(k)$ is $\widetilde{\mathbf{x}}_{i}^{c}(k)$, i.e. the extended Chebyshev center of the $i$-th agent's Voronoi cell. The elements of $\boldsymbol{Q}, \boldsymbol{R}_{1}$ and $\boldsymbol{R}_{2}$ are chosen in order to prioritize the terms as follows: reach the agent's designed position at constant altitude, limit the input signal amplitude and its variations.

In (23f), $\mathbb{V}_{i}^{\lambda}(k)=\mathbf{c}_{i}^{c}(k) \oplus \lambda\left(\mathbb{V}_{i}(k) \oplus\left\{-\mathbf{c}_{i}^{c}(k)\right\}\right)$, where $\mathbf{c}_{i}^{c}(k)$ is the Chebyshev center of $\mathbb{V}_{i}(k)$ and $0 \leq \lambda<1$. The set $\mathbb{V}_{i}^{\lambda}(k)$ is then a contracted version of $\mathbb{V}_{i}(k)$ centered on its Chebyshev center. This constraint ensures that $\mathbb{V}_{i}^{\lambda}(k)$ is a controlled $\lambda$-contractive set [11].

\section{B. Formation reconfiguration in case of outgoing agents}

This part proposes a novel predictive control strategy for the formation reconfiguration once non-cooperating agents are detected (e.g. faulty agents or agents having to leave the formation for other purposes).

The control signal $\widetilde{\mathbf{u}}_{i}(k)$ is obtained by solving the optimization problem with the cost function (23a) under the constraints (23b), (23c) (where $\boldsymbol{G}$ can be modified in case of a faulty agent), (23d) (where $\mathbb{W}$ is replaced by $\mathbb{X}$ ) and (23e).

The objective point $\widetilde{\mathbf{x}}_{i}^{\text {obj }}$ is a point outside the workspace $\mathbb{W}$ that the outgoing agent can reach given the potential loss of effectiveness in one or more of its actuators.

Assumption 6. The communication between the agents is perfect with no package loss or communication delay.

When an agent $i_{n c} \in \mathscr{N}_{f}$ happens to be non-cooperating, it broadcasts its position and its objective $\widetilde{\mathbf{x}}_{i_{n c}}^{\text {obj }}$ to the other agents $i \in \mathscr{N}_{f} \backslash\left\{i_{n c}\right\}$ in the formation. These points define a hyperplane $\mathscr{H}$ in $\mathscr{W}$. These agents will change $\widetilde{\mathbf{x}}_{i}^{\text {obj }}$ to their neighbors' barycenter in (23). Based on the definition of $\mathscr{H}$, the agents remaining in formation will compute their neighbors' barycenter.
Definition 1. For an agent $i \in \mathscr{N}_{f}$, a neighbor is either an agent having a Voronoi cell contiguous to $\mathbb{V}_{i}$ or a vertex of $\mathbb{V}_{i}$ lying on the border of $\mathscr{W}$.

Consider one cooperating agent $i \in \mathscr{N}_{f} \backslash\left\{i_{n c}\right\}$ and one of its neighbors $j \in \mathscr{N}_{i}$. If $\mathbf{x}_{i}^{r}$ and $\mathbf{x}_{j}^{r}$ lie in the same halfspace and the distance between $\mathbf{x}_{j}^{r}$ and $\mathscr{H}$ is greater than the distance between $\mathbf{x}_{i}^{r}$ and $\mathscr{H}$, the weight of agent $j$ is set to $\kappa w$, with $\kappa>1$ and $w>0$. Else, the agent $j$ 's weight is set to $w$. The extended barycenter is then $\widetilde{\mathbf{x}}_{i}^{\mathrm{bar}}=\left[\begin{array}{ll}\mathbf{c}^{\mathrm{bar}} & \mathbf{0}_{1 \times 10}\end{array}\right]$, where $\mathbf{c}^{\text {bar }}$ is the weighted average of the $\mathbf{x}_{j}^{r}$, with $j \in \mathscr{N}_{i}$. By tracking this point, the remaining agents will move away from the leaving agent by construction.

The non-cooperating agent then leaves the workspace $\mathbb{W}$ relying on the problem described in this section. In the meantime, the remaining agents track their neighbors' barycenter by solving the problem (23) with $\widetilde{\mathbf{x}}_{i}^{\text {obj }}=\widetilde{\mathbf{x}}_{i}^{\text {bar }}$.

\section{Formation reconfiguration in case of incoming agents}

This part proposes a control algorithm allowing healthy agents from $\mathbb{Y}$ (but not in $\mathbb{W}$ ) to join in the formation. To do that, the control signal $\widetilde{\mathbf{u}}_{i}(k)$ for the agent joining in the formation is obtained by solving the same optimization problem as in Section IV-B.

The objective point $\widetilde{\mathbf{x}}_{i}^{\text {obj }}=\left[\begin{array}{ll}\mathbf{c}_{i}^{\text {ref }} & \mathbf{0}_{1 \times 10}\end{array}\right]$, where $\mathbf{c}_{i}^{\text {ref }} \in \mathscr{W}$ is the closest point to the incoming agent inside $\mathbb{W}$ in terms of the Euclidean norm. When the incoming agent is inside $\mathbb{W}$, it changes its control algorithm to the one defined in Section IV-A to participate in the formation deployment.

\section{Simulations And Results}

\section{A. Simulation scenarios}

The control algorithms presented in this paper are tested in simulation with MATLAB with MPT3.0 [26] and solvers generated using CVXGEN [27].

The restricted workspace $\mathscr{W}$ is a bounded square of side length $20 \mathrm{~m}$ centered at $(0,0)$. For all the optimization problems, the prediction horizon is set to $N_{p}=10$ in order to be large enough for the constraints to be satisfied but also small enough for the target point (assumed to be fixed over the prediction horizon) to be close to its real position at the end of the horizon.

First scenario. For the formation deployment problem, seven UAVs are considered with the nonlinear dynamics described in Section II-B. They rely on the control algorithm from Section IV-A and the linear dynamics from Section II-C. The agents start in a hovering state at random locations in $\mathscr{W}$, their initial conditions being set to $\mathbf{x}_{i}^{\text {init }}=$ $\left[\begin{array}{llll}x_{i} & y_{i} & \bar{z} & \mathbf{0}_{1 \times 9}\end{array}\right]^{\top}$. They track the Chebyshev center of their Voronoi cell to converge to a Chebyshev configuration.

Second scenario. Once the seven agents are deployed, at $t=20 \mathrm{~s}$, a loss of $40 \%$ of its effectiveness is injected to the rear rotor of one of the agents. The outgoing agent leaves the formation using the algorithm described in Section IV-B. The other agents rally their neighbors' barycenter while the faulty agent is inside the workspace. When the faulty agent leaves the workplace, the remaining agents reconfigure for an optimal distribution within the workspace. 


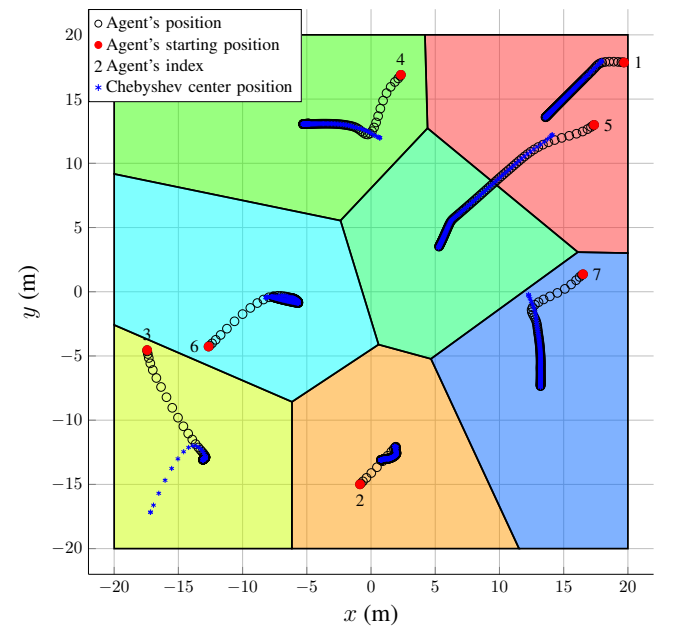

Fig. 2. Formation deployment over time in nominal case lying in the Voronoi tessellation of $\mathscr{W}$ at $t=20 \mathrm{~s}$.

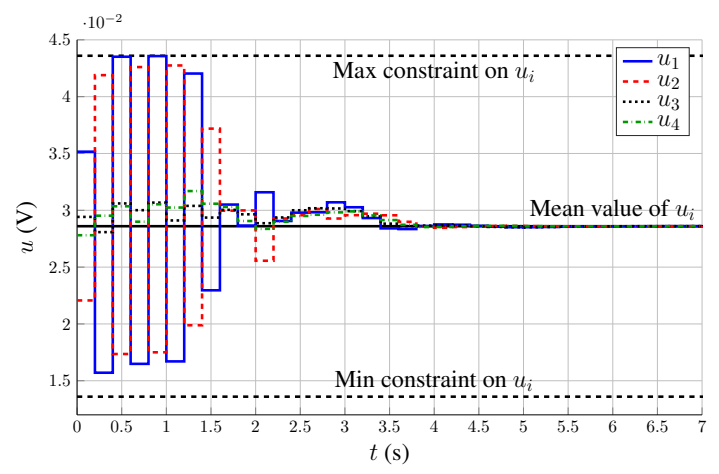

Fig. 3. Control signals of agent 3 during formation deployment.

Third scenario. The seven UAVs start in a Chebyshev configuration and three incoming agents outside the workspace $\mathbb{W}$ join in the formation. The incoming agents rely on the algorithm described in Section IV-C to join in the formation.

The main objectives of the agents are to reach the Chebyshev center of their cell and to remain at a constant altitude with a null yaw angle. Thus $Q$ is chosen to be $Q=$ $\operatorname{diag}(10,10,100,1,1,100,1,1,100,1,1,100)$. The weights $\boldsymbol{R}_{1}$ and $\boldsymbol{R}_{2}$ are set to $\boldsymbol{R}_{1}=1000 \boldsymbol{I}_{4}$ and $\boldsymbol{R}_{2}=10 \boldsymbol{R}_{1}$ to follow these objectives while keeping an input signal $\left|\widetilde{u}_{i}^{j}\right| \leq 0.015 \mathrm{~V}, i \in\{1, \ldots, 4\}, j \in \mathscr{N}=\{1, \ldots, 7\}$. These weights are the same for the three algorithms. For the computation of the barycenter, the weights $w=1$ and $\kappa=3$ are considered.

\section{B. Analysis of the results}

First scenario. The initial positions of the UAV agents are represented by a filled red circle on Fig. 2. As shown on Fig. 2, all the agents converge to their objective in a finite time. The control signals of one of the agents (agent 3 from Fig. 2) are presented on Fig. 3. These signals oscillate around the equilibrium point $\bar{u}=\frac{m g}{4 K}$. The signals $u_{1}$ and $u_{2}$ have a larger amplitude than the signals $u_{3}$ and $u_{4}$ in the case of agent 3 because its movement is mainly along the $\mathbf{y}$ axis.

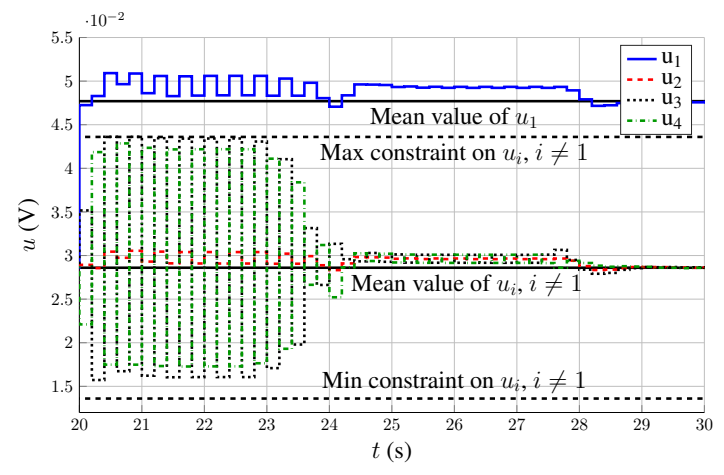

Fig. 4. Control signals of agent 5 from fault detection onward.

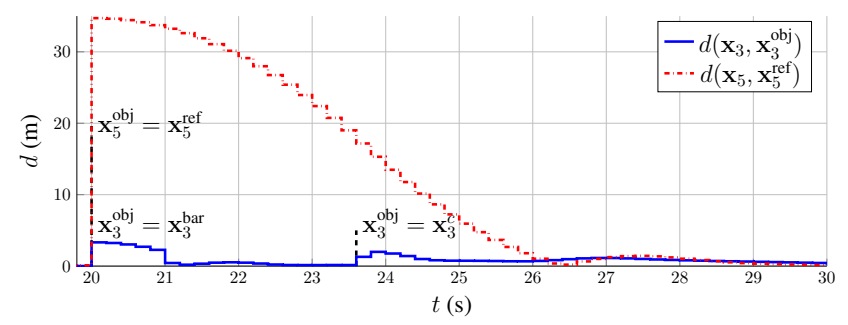

Fig. 5. Distance of the first two coordinates of agent 5 and agent 3 to their objective with respect to time.

Second scenario. A loss of $40 \%$ of its effectiveness is injected to the first rotor of agent 5. The value of $\bar{u}$ and the constraint on $u_{1}$ are then increased by $66.6 \%$ compared to their nominal values in order to compensate the loss of effectiveness. The agent must take the shortest way out of the formation. Its target point is then set to $\mathbf{x}_{5}^{\text {ref }}=\left[\begin{array}{llll}40 & y(t=20 \mathrm{~s}) & \bar{z} & \mathbf{0}_{1 \times 9}\end{array}\right]^{\top}$. The control signals for the rotors of agent 5 are presented on Fig. 4.

On Fig. 5, the objectives of agent 3 and agent 5 are changed according to the procedure described in Section IV-B. The distances from the agents to their objectives converge to 0 and the outgoing agent successfully leaves the formation. When the outgoing agent has left, the formation is properly reconfigured.

A video showing the simulation of the first two scenarios is available at https://youtu.be/LhfnvSxrmwI.

Third scenario. The seven agents start from the same positions as in the first and second scenarios. At $t=0$ $\mathrm{s}$, three agents join in the multi-agent system with initial state vectors $\mathbf{x}_{8}^{\text {init }}=\left[\begin{array}{llll}-10 & 30 & \bar{z} & \mathbf{0}_{1 \times 9}\end{array}\right]^{\top}, \mathbf{x}_{9}^{\text {init }}=$ $\left[\begin{array}{llll}0 & 30 & \bar{z} & \mathbf{0}_{1 \times 9}\end{array}\right]^{\top}, \mathbf{x}_{10}^{\text {init }}=\left[\begin{array}{llll}10 & 30 & \bar{z} & \mathbf{0}_{1 \times 9}\end{array}\right]^{\top}$. These three agents join in the formation while the seven others still deploy relying on the formation deployment algorithm. The control signals for agent 9 are presented on Fig. 6 .

At $t=3.4 \mathrm{~s}$, agents 8,9 and 10 enter the workspace $\mathbb{W}$. They then start tracking the Chebyshev center of their associated Voronoi cell. On Fig. 7, agent 9 converges towards its objective point inside $\mathbb{W}$ as well as the other agents.

The simulation results of the third scenario are available at https://youtu.be/ecUbW19XxkA. 


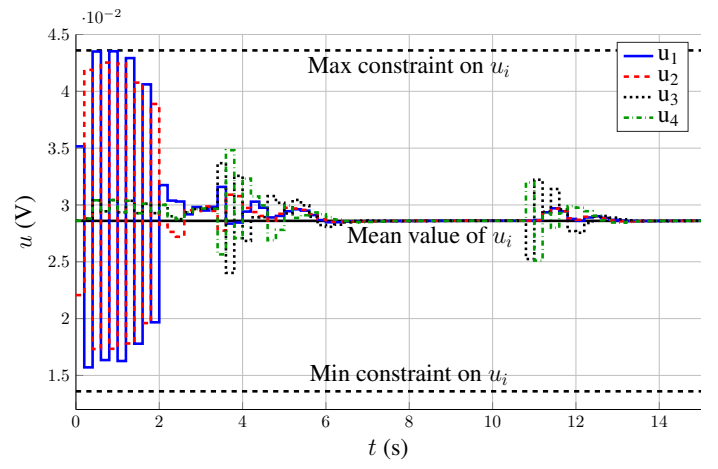

Fig. 6. Control signals of agent 9 while it joins the formation until the end of formation reconfiguration.

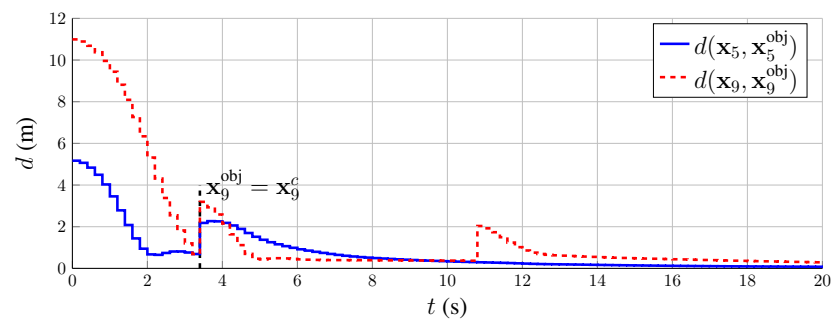

Fig. 7. Distance of the first two coordinates of agent 9 and agent 5 to their objective with respect to time.

\section{CONCLUSION}

This paper presents decentralized linear model predictive control techniques for quadrotor unmanned aerial vehicles (UAVs) formation deployment and reconfiguration over a convex bounded area based on dynamic Voronoi tessellation. Using this control approach: 1) the formation is able to deploy in a static Chebyshev configuration over the area; 2) non-cooperating UAVs can leave the formation or recovered/healthy UAVs can join in the formation while avoiding collisions. Three simulation scenarios have been proposed to test the formation deployment and the formation reconfiguration in case of an outgoing faulty UAV and in case of three incoming healthy UAVs.

In our future work, the control algorithms will be applied to an experimental testbed of UAVs available in the Networked Autonomous Vehicles Lab in Concordia University. Moreover, different types of faults will be investigated such as sensor faults or communication delays/loss. Finally, the shape and size of the formation's workspace could also be time-varying depending on the needs of the mission.

\section{ACKNOWLEDGMENT}

The authors would like to acknowledge support for this work from the LIA of the CNRS on Information, Learning and Control and Natural Sciences and Engineering Research Council of Canada.

\section{REFERENCES}

[1] A. S. Laliberte and A. Rango, "Texture and scale in object-based analysis of subdecimeter resolution unmanned aerial vehicle (UAV) imagery," IEEE Transactions on Geoscience and Remote Sensing, vol. 47, no. 3, pp. 761-770, 2009.
[2] D. W. Casbeer, R. W. Beard, T. W. McLain, S.-M. Li, and R. K. Mehra, "Forest fire monitoring with multiple small UAVs," in American Control Conference, 2005, pp. 3530-3535.

[3] F. Nex and F. Remondino, "UAV for 3D mapping applications: A review," Applied Geomatics, vol. 6, no. 1, pp. 1-15, 2014.

[4] T. Tomic, K. Schmid, P. Lutz, A. Domel, M. Kassecker, E. Mair, I. L. Grixa, F. Ruess, M. Suppa, and D. Burschka, "Toward a fully autonomous UAV: Research platform for indoor and outdoor urban search and rescue," IEEE Robotics \& Automation Magazine, vol. 19, no. 3, pp. 46-56, 2012.

[5] R. Lozano, Unmanned aerial vehicles: Embedded control. John Wiley \& Sons, 2013.

[6] F. Rinaldi, S. Chiesa, and F. Quagliotti, "Linear quadratic control for quadrotors UAVs dynamics and formation flight," Journal of Intelligent \& Robotic Systems, vol. 70, pp. 203-220, 2013.

[7] D. M. Stipanović, G. Inalhan, R. Teo, and C. J. Tomlin, "Decentralized overlapping control of a formation of unmanned aerial vehicles," Automatica, vol. 40, no. 8, pp. 1285-1296, 2004.

[8] D. Galzi and Y. Shtessel, "UAV formations control using high order sliding modes," in American Control Conference, 2006, pp. 42494254.

[9] A. Bemporad and C. Rocchi, "Decentralized linear time-varying model predictive control of a formation of unmanned aerial vehicles," in IEEE 50th Conference on Decision and Control and European Control Conference, 2011, pp. 7488-7493.

[10] Y. Zhang and J. Jiang, "Bibliographical review on reconfigurable faulttolerant control systems," Annual Reviews in Control, vol. 32, no. 2, pp. 229-252, 2008.

[11] F. Blanchini and S. Miani, Set-theoretic methods in control. Springer, 2007.

[12] R. M. Murray, "Recent research in cooperative control of multivehicle systems," Journal of Dynamic Systems, Measurement, and Control, vol. 129 , no. 5, pp. 571-583, 2007.

[13] M. T. Nguyen, C. Stoica Maniu, and S. Olaru, "Optimization-based control for multi-agent deployment via dynamic Voronoi partition," 20th IFAC World Congress, vol. 50, no. 1, pp. 1828-1833, 2017.

[14] J. Cortes, S. Martinez, T. Karatas, and F. Bullo, "Coverage control for mobile sensing networks," IEEE Transactions on Robotics and Automation, vol. 20, no. 2, pp. 243-255, 2004.

[15] F. Sharifi, A. Chamseddine, H. Mahboubi, Y. Zhang, and A. G Aghdam, "A distributed deployment strategy for a network of cooperative autonomous vehicles," IEEE Transactions on Control Systems Technology, vol. 23, no. 2, pp. 737-745, 2015.

[16] G. Voronoï, "Nouvelles applications des paramètres continus à la théorie des formes quadratiques," Journal für die reine und angewandte Mathematik, vol. 134, pp. 198-287, 1908.

[17] J. M. Hendrickx and S. Martin, "Open multi-agent systems: Gossiping with random arrivals and departures," in IEEE 56th Conference on Decision and Control, 2017, pp. 763-768.

[18] S. Bouabdallah, P. Murrieri, and R. Siegwart, "Design and control of an indoor micro quadrotor," in IEEE International Conference on Robotics and Automation, vol. 5, 2004, pp. 4393-4398.

[19] L.-C. Lai, C.-C. Yang, and C.-J. Wu, "Time-optimal control of a hovering quad-rotor helicopter," Journal of Intelligent \& Robotic Systems, vol. 45, pp. 115-135, 2006.

[20] M. Abdolhosseini, Y. Zhang, and C. A. Rabbath, "An efficient model predictive control scheme for an unmanned quadrotor helicopter," Journal of Intelligent \& Robotic Systems, vol. 70, pp. 27-38, 2013.

[21] H. Eggleston, Convexity. Cambridge University Press, 1958.

[22] A. Schrijver, Theory of linear and integer programming. John Wiley \& Sons, 1998.

[23] S. Boyd and L. Vandenberghe, Convex optimization. Cambridge University Press, 2004.

[24] M. T. Nguyen, "Safe predictive control for multi-agent dynamical systems," Theses, Université Paris-Saclay, 2016.

[25] A. Bemporad, M. Morari, V. Dua, and E. N. Pistikopoulos, "The explicit linear quadratic regulator for constrained systems," Automatica, vol. 38, no. 1, pp. 3-20, 2002.

[26] M. Herceg, M. Kvasnica, C. N. Jones, and M. Morari, "Multiparametric toolbox 3.0," in European Control Conference, 2013, pp. $502-510$.

[27] J. Mattingley and S. Boyd, "CVXGEN: A code generator for embedded convex optimization," Optimization and Engineering, vol. 13, no. 1, pp. 1-27, 2012. 